\title{
$d$ - $d$ Spectra of Transition-Metal Carbodiimides and Hydrocyanamides as Derived from Many-Particle Effective Hamiltonian Calculations
}

\author{
Andrei L. Tchougréeff ${ }^{1,2}$ and Richard Dronskowski ${ }^{1}$
}

January 29, 2011

\author{
${ }^{1}$ Institut für Anorganische Chemie, RWTH Aachen University, Landoltweg 1, 52056 \\ Aachen, Germany; \\ ${ }^{2}$ Poncelet Laboratory, Independent University of Moscow, Bolshoy Vlasyevskiy Pereulok \\ 11, 119002, Moscow, Russia
}

\begin{abstract}
We apply the local many-particle method of the Effective Hamiltonian of Crystal Field (EHCF) to analyze the magnetic ground state and the low-energy excitation spectra of the transition-metal carbodiimides of the general formula $M N C N$ with $M$ $=\mathrm{Fe}-\mathrm{Ni}$. Experimentally these materials represent a uniform group of (high-spin) antiferromagnetic, optically transparent, colored insulators with absorption lines in the visible spectrum. These findings are fully supported by the EHCF numerical modelling. In all three cases we arrive at high-spin ground states in agreement with the results of previous magnetic measurements as well as the presence of the $d$ - $d$ intrashell transitions for the visible absorption spectra. Remarkably enough, the EHCF approach resolves the controversial case of FeNCN which was earlier predicted to be metallic by density-functional theory even when including explicit electronic correlation $(\mathrm{GGA}+U)$. We also address the ground state and the lowenergy excitation spectra of the transition-metal hydrocyanamides of the general formula $M(\mathrm{NCNH})_{2}$ with $M=\mathrm{Fe}-\mathrm{Ni}$, another uniform group of optically transparent colored insulators. EHCF also arrives at high-spin ground states and visible $d$ - $d$ intrashell transitions.
\end{abstract}

\section{Introduction}

Materials traditionally used for the fabrication of magnetic memory devices are typically metal oxide-based systems. Their physical properties are governed by effective local momenta residing in the $d$-shells of the transition-metal cations whose interactions are 
mediated by oxide $\left(\mathrm{O}^{2-}\right)$ dianions. Organometallic chemistry provides routes to alternative materials, i.e., coordination polymers or "metal-organic" frameworks [1]. To replace $\mathrm{O}^{2-}$, a natural way is to use quasi-molecular bridges with nitrogen atoms. Our group has recently synthesized and characterized the antiferromagnetic $3 d$ carbodiimides [2-4] incorporating $3 d$ cations connected by $\mathrm{NCN}^{2-}$ dianions as $\mathrm{O}^{2-}$ substitutes. Antiferromagnetic interactions are characteristic for all family members, and the archetypal phase MnNCN serves as a perfect example. The electrons residing in the partially filled $3 d$ shells of $\mathrm{Mn}^{2+}$ in $\mathrm{MnNCN}$ are clearly localized which manifests in its optical transparency and insulating electrical behavior. Similarly, all other $M$ NCN compounds with $M=\mathrm{Fe}-\mathrm{Ni}$ represent a uniform family being optically transparent and electrically insulating as well $[3,4]$. We have studied the electronic structures and magnetic properties of $\operatorname{MNCN}(M=\mathrm{Fe}$, Co and $\mathrm{Ni}$ ) [5] by density-functional theory including explicit electronic correlation through an ad hoc Coulomb potential $(\mathrm{GGA}+U)$ in order to establish analogies with the corresponding oxides - a benchmark for the DFT-based methods as applied to solid phases with open $d$-shells [6]. The results conform with the picture that CoNCN and NiNCN are type-II antiferromagnetic insulators, that is, intralayer ferromagnetic and interlayer antiferromagnetic ordering of atomic spin densities residing on metal atoms and wide gaps in the spectra of one-electronic band states. Thus, the $M$ NCN phases with $M=\mathrm{Ni}$, Co resemble the corresponding $M O$ monoxides with respect to their optical and transport properties. By contrast, FeNCN remains, according to the GGA $+U$ calculation (semi)metallic, even upon applying a strong Coulomb correlation potential. This points towards a serious density-functional theory problem.

In order to come up with a better theoretical modelling we now focus on the manyparticle/local character as opposed to the single-particle/delocalized DFT picture. Here we present the required many-particle numerical study. In principle, the general tool for such tasks is provided by the dynamic mean-field theory (DMFT) [7]. Its applications to real-world materials are, however, restricted by the high computational costs. The alternative effective Hamiltonian crystal field (EHCF) method [8] may be seen as a specialized version of DMFT suitable for describing the situation with well-defined $d$-shells and for the optical range of energies. The variance with DMFT (not mentioning technical details addressed below) is that the electron transfers from and to the impurity states of DMFT are converted in the operators renormalizing the impurity one-electron Hamiltonian. The impurity problem is then solved using full configuration interaction rather than by the 
quantum Monte-Carlo method. By doing so the EHCF method has recently allowed us to obtain detailed and precise description of the local excitation spectrum of the $d$-shells in archetypal MnNCN [9].

The "organic" path of materials design with predefined properties has the advantage of chemical modification of the bridges, the simplest one being protonation. Thus, we have synthesized and characterized hydrocyanamides $M(\mathrm{NCNH})_{2}(M=\mathrm{Fe}-\mathrm{Ni})[10]$ as hydrated analogues of $M$ NCN. Since our previous studies of electronic structures and magnetic properties of $M \mathrm{NCN}(M=\mathrm{Fe}, \mathrm{Co}$ and $\mathrm{Ni}$ ) [5,9] have shown that DFT approaches may suffer from irregularly appearing qualitative errors, we also apply the EHCF technique to the $M(\mathrm{NCNH})_{2}$ family.

\section{EHCF Method}

The EHCF method is based on the simple physical picture that the electronic states of a transition-metal compound with partially filled $d$-shells can be presented [11] as band states of an insulator (such as $\mathrm{MgO}$ ) supplied with the local $d$-multiplets. Their excitations determine the observed optical spectra and naturally conform to the qualitative spectral picture $[12,13]$. Just like in the oxides the $\mathrm{NCN}^{2-}$ dianions span the band states which are complemented by the local states of the corresponding $d$-shells. In contrast with the one-electron/delocalized-bands paradigm underlying DFT the $d$-states of EHCF remain local, but are many-particle correlated states: they remain partially filled, but do not produce any noticeable electric conductivity.

Since the characteristic energy of the EHCF method is that of the optical transitions, the wave-function formalism may apply, in variance with the general DMFT where the temperature Green's functions are used [7]. The electronic wave function formalizing the physical requirements corresponds to a fixed number of electrons in the $d$-shell as a zeroorder approximation. The interactions responsible for electron transfer between the $d$ shell and the bands of the insulator they are immersed to are considered as perturbations. Following the standard semiempirical setting we restrict the AO basis for all atoms by the valence orbitals. These are then separated into two subsets from which one (the $d$-system) contains $3 d$-orbitals of the transition-metal atom, and another (the ligand subsystem, also called the $l$-system) contains $4 s$ - and $4 p$-orbitals of the transition-metal atom and the valence AOs of all ligand atoms. We consider only those systems whose excitation energies in the $l$-system are by far larger than those in the $d$-shell of the metal atom (the 
effect of excitations in the $l$-system has been considered in detail [14]).

The electronic wave function for the $n$-th state is written as the antisymmetrized product of the wave functions of the electron groups introduced above:

$$
\Psi_{n}=\Phi_{d}^{(n)} \wedge \Phi_{l}
$$

The low-energy $d$ - $d$-spectrum of the whole system is described by the Hamiltonian

$$
H=H_{d}+H_{l}+H_{c}+H_{r}
$$

where $H_{d}$ describes $d$-electrons, $H_{l}$ the ligand system, $H_{c}$ the Coulomb interaction, and $H_{r}$ the resonance interaction. The effective Hamiltonian for the $d$-shell only reads

$$
H_{d}^{\mathrm{eff}}=\sum_{\mu \nu \sigma} U_{\mu \nu}^{\mathrm{eff}} d_{\mu \sigma}^{+} d_{\nu \sigma}+\frac{1}{2} \sum_{\mu \nu \rho \eta} \sum_{\sigma \tau}(\mu \nu \mid \rho \eta) d_{\mu \sigma}^{+} d_{\rho \tau}^{+} d_{\eta \tau} d_{\nu \sigma}
$$

where the $d$-electron Coulomb interaction term is inherited from the free ion, and the effective core parameters $U_{\mu \nu}^{\text {eff }}$ contain contributions from the Coulomb and the resonance interaction between the $d$ - and $l$-systems:

$$
U_{\mu \nu}^{\mathrm{eff}}=\delta_{\mu \nu} U_{d d}+W_{\mu \nu}^{\mathrm{atom}}+W_{\mu \nu}^{\text {field }}+W_{\mu \nu}^{\mathrm{cov}}
$$

where

$$
W_{\mu \nu}^{\text {atom }}=\delta_{\mu \nu}\left(\sum_{\alpha \in s, p} g_{\mu \alpha} P_{\alpha \alpha}\right)
$$

is the repulsion of electrons in the $d$-shell from those in the $4 s$ - and $4 p$-AOs of the metal expressed through their densities $P_{\alpha \alpha}$ and

$$
W_{\mu \nu}^{\text {field }}=\sum_{L} Q_{L} V_{\mu \nu}^{L}
$$

is the Coulomb interaction of the $d$-electrons with the net charges $Q_{L}$ on the ligand atoms, having the standard crystal-field theory form [12] of the matrix elements $V_{\mu \nu}^{L}$. The covalence part

$$
W_{\mu \nu}^{\mathrm{cov}}=-\sum_{i} \beta_{\mu i} \beta_{\nu i}\left(\frac{1-n_{i}}{\Delta E_{d i}}-\frac{n_{i}}{\Delta E_{i d}}\right)
$$

comes from the resonance interaction between the $d$ - and $l$-systems. This term precisely corresponds to the hybridization term in the Anderson impurity model as employed within DMFT [7]. Here $n_{i}$ is the occupation number of the $i$-th $l$-MO $\left(n_{i}=0\right.$ or 1$)$, and $\Delta E_{d i}$ 
$\left(\Delta E_{i d}\right)$ is the energy necessary to transfer an electron from the $d$-shell (from the $i$-th $l$-MO) to the $i$-th $l$-MO (to the $d$-shell).

Within EHCF [8] the $l$-system is described by a single Slater determinant $\Phi_{l}$ (formed by bona fide approximations to the valence band state) which has to be obtained from a Hartree-Fock-Roothaan (HFR) procedure. Solving the HFR problem for the $l$-system yields the one-electron density matrix $P_{\alpha \beta}$, orbital energies $\varepsilon_{i}$, and the MO-LCAO coefficients $c_{i \alpha}$. These quantities completely define the electronic structure of the $l$-system and are used to calculate the effective Hamiltonian (eq. 3) by eqs. 5-7. Further details of the method's implementation can be found in the literature $[8,15,16]$.

The problem of semiempirical modeling is seen somewhat different from that of standard procedures including DFT. The EHCF method was parameterized for calculations of various isolated complexes of the $3 d$ row, with mono- and polyatomic ligands. The parameters scaling the resonance interactions between the $d$-shells and the environment with donor atoms $\mathrm{N}, \mathrm{C}, \mathrm{O}, \mathrm{F}, \mathrm{Cl}$ and for doubly and triply charged ions of $\mathrm{V}, \mathrm{Cr}, \mathrm{Mn}, \mathrm{Fe}$, $\mathrm{Co}, \mathrm{Ni}$ and $\mathrm{Cu}$ have been fitted [8,15-17]. In contrast with the angular overlap model [18], all the necessary elements of the theory are calculated rather than individually fitted to reproduce the observed spectra. The parameters do not depend on structural details of the ligands. Further evaluations $[19,20]$ have shown the applicability of these for calculating the electronic structure and spectra of numerous complexes of divalent cations, merely based on the CNDO parameterization for the $l$-system. In all calculations the experimental multiplicity (spin) and spatial symmetry of the corresponding ground states was correctly reproduced.

The above considerations only slightly depend on the difference between the molecular and solid-state EHCF settings. We have shown $[21,22]$ that the use of adequately sized clusters is necessary for calculating the atomic effective charges as well as the corresponding orbital energies and one-electron states with sufficient accuracy.

\section{Results and Discussion}

The calculations have been performed for the 29-atomic $\left[M_{5}(\mathrm{NCN})_{8}\right]^{6-}$ clusters of the $M \mathrm{NCN}$ and for the neutral 81-atomic $\left[M_{7}(\mathrm{NCNH})_{18}\right]$ clusters of the $M(\mathrm{NCNH})_{2}$ phases cut from their experimental crystal structures as shown in Figures 1 and 2, respectively. For $M \mathrm{NCN}$, the transition-metal cation is octahedrally coordinated by nitrogen atoms. The carbodiimide unit, however, is found in a trigonal prism by $M$ atoms, thus resembling 
the $[\mathrm{NiAs}]$ motif.

The transition-metal ions for which the excitation spectra were calculated were placed in the geometric center of the cluster. No additional parameterization as compared to $[19,20]$ was performed. For the case of $M N C N$ we could not reach convergence of the HFR procedure for larger clusters. Nonetheless, as shown before $[9,21,22]$ the used cluster size is sufficient for obtaining reliable spectral results. Similarly, the nonvanishing cluster charge may lead only to a general shift of the energy levels and does not affect the energy differences, that is, the transition energies. The results are given in Table 1. In all cases the ground state corresponds to the high-spin configuration characteristic for weak-field ligands with nitrogen donor atoms, as expected. Like in the case of MnNCN [9], the actual symmetry of the $M \mathrm{~N}_{6}$ chromophores in $M \mathrm{NCN}$ is lower than that of the ideal octahedron. Both the trigonal distortion of the chromophore due the spatial arrangement of the nonspherical (in fact, rod-like) $\mathrm{NCN}^{2-}$ anions and the local distortions of the $\mathrm{M}-\mathrm{N}$ bond lengths and $\mathrm{N}-\mathrm{M}-\mathrm{N}$ angles contribute to this symmetry lowering. In any case the splittings within approximate octahedral manifolds of states largely do not exceed 500 $\mathrm{cm}^{-1}$.

A comparison of the spin moments of the calculated ground states of the $M \mathrm{NCN}$ compounds with the corresponding measured values of the effective magnetic moments $[3,4]$ requires a special procedure similar to that described before [5]. We will briefly describe the related issues. Because the magnetic properties of the archetypal phase MnNCN [2] are characteristic for a weak-field (that is, $S=5 / 2$ high-spin) scenario, the first estimate for the observed magnetic moments of FeNCN $(S=2)$, CoNCN $(S=3 / 2)$ and NiNCN $(S=1)$ may safely be based on the spin-only value

$$
\mu_{\mathrm{eff}}^{\mathrm{spin}}=2 \sqrt{S(S+1)} \mu_{\mathrm{B}}
$$

where 2 stands for the approximate value of the electronic $g$-factor. Thus, the pure spin moments arrive at the familiar 4.90, 3.87, and $2.83 \mu_{\mathrm{B}}$ for divalent Fe, Co, and $\mathrm{Ni}$, respectively. In addition, these values will be further enhanced due to spin-orbit interaction in the weak-field (high-spin) manifolds such that effective moments of 5.0-5.5, 4.0-5.0, and 3.0-3.5 $\mu_{\mathrm{B}}$ are to be expected, at least for magnetically dilute $\mathrm{Fe}$, Co and Ni compounds [26].

Nonetheless, the entire $M \mathrm{NCN}$ series members can by no means be considered as magnetically diluted; on the contrary, antiferromagnetic ground states with rather high Néel temperatures have been reported $[3,4]$. Unfortunately, the thermal instability of 
these materials made it necessary to measure the $T$-dependent effective magnetic moments $\mu_{\text {eff }}^{*} \sim \sqrt{\chi_{m} T}$ solely in the paramagnetic regimes close to (that is, slightly above) the Néel points of FeNCN, CoNCN and NiNCN, not at very high temperatures. Thus, these moments are still strongly contaminated by antiferromagnetic correlations, and these scale down [5] the true (high-temperature) effective moments $\mu_{\text {eff }}$ by a factor of $\sqrt{T /\left(T+\left|\theta_{\mathrm{CW}}\right|\right)}<1$.

Because the magnitudes of $\theta_{\mathrm{CW}}$ were not extractable from the magnetic susceptibilities, they had to be replaced by the molecular-field estimates, expressed by the exchange parameters $J_{1}$ and $J_{2}$. The conversion coefficients are

$$
\mu_{\mathrm{eff}} / \mu_{\mathrm{eff}}^{*}=\sqrt{\frac{2}{1-\left(J_{2} / 3 J_{1}\right)}}>1,
$$

provided the antiferromagnetically contaminated $\mu_{\mathrm{eff}}^{*}$ are measured precisely at the Néel temperature [5]. Using the estimated $J_{1}$ and $J_{2}$ values, the conversion coefficients are 1.322 for NiNCN and 1.368 for CoNCN. As a result, the experimental effective moments as cleaned from the antiferromagnetic correlations are $2.38 \mu_{\mathrm{B}}$ for $\mathrm{NiNCN}$ and $4.24 \mu_{\mathrm{B}}$ for CoNCN. The former value is in a reasonable agreement with the spin-only value whereas the latter fairly conforms with the EHCF-based prediction of $4.58 \mu_{\mathrm{B}}$. It has been obtained following the published prescriptions [25] including the spin-orbit interaction constant $\zeta$ of $456 \mathrm{~cm}^{-1}$ and other necessary values taken form Table 1 . The spin-orbit interaction further enhances [25] the spin-only magnetic moment of NiNCN by a factor of $\sqrt{1+\frac{4 \zeta}{\Delta}} \sim 1.17$, where $\Delta$ stands for the energy of the lowest excited $T$-state of the $d^{8}$-shell of $\mathrm{Ni}^{2+}$. For the moment, we cannot explain the relatively small experimental value of NiNCN as derived from the magnetic measurements.

For the case of FeNCN, the $J_{1}$ and $J_{2}$ estimates [5] are useless because the DFT ground state does not represent an antiferromagnetic insulator. Based on the similarity of structure and magnetic behavior of FeNCN to the other two phases, we simply adopt the same conversion coefficient as for CoNCN. The cleaned momentum of FeNCN arrives at $5.33 \mu_{\mathrm{B}}$, in reasonable agreement with $5.48 \mu_{\mathrm{B}}$ as predicted by EHCF. It includes the spin-orbit interaction [25] and an interaction parameter $\zeta$ of $410 \mathrm{~cm}^{-1}$.

Summarizing, we conclude that the EHCF-predicted spectra as well as the the EHCFderived effective magnetic moments fairly conform with the entire body of experimental data. Remarkably enough, the case which turned pathological for the GGA $+U$ approach (FeNCN) is unequivocally ascribed by the EHCF method to the same category as its 
CoNCN and NiNCN analogs. To round up the discussion, we note that the present analysis of magnetic data is in variance with a preceding one [5] in which our aim was to compare the same experimental data (effective magnetic moments measured in the paramagnetic regimes closely above the Néel temperatures [3,4]) with DFT-derived magnetizations. The latter correspond to saturated magnetic moments in the ordered zerotemperature phases. Because the saturation momenta scale as $\mu_{\text {sat }} \sim S$ rather than as $\sim \sqrt{S(S+1)}$, they must be smaller than the high-temperature moments cleaned from antiferromagnetic correlations:

$$
\mu_{\mathrm{eff}}=\sqrt{(1+1 / S)} \mu_{\mathrm{sat}}
$$

The spin-dependent factor takes into account the quantum fluctuations.

With respect to the hydrocyanamides, an unequivocal experimental value of an effective magnetic momentum, $4.9 \mu_{\mathrm{B}}$, is only available for $\mathrm{Fe}(\mathrm{NCNH})_{2}$ [4]. This is in good agreement with the spin-only value and a reasonable allowance for the magnetic coupling as in the $M N C N$ case. The magnetic behavior of $\mathrm{Co}(\mathrm{NCNH})_{2}$ and $\mathrm{Ni}(\mathrm{NCNH})_{2}$ can be roughly described as ferromagnetic [24] with Curie temperatures of ca. 10 and $30 \mathrm{~K}$, respectively. The reciprocal magnetic susceptibility is almost ideally linear for $\mathrm{Ni}(\mathrm{NCNH})_{2}$ so that a reliable experimental value of the high-temperature effective momentum yields $3.12 \mu_{\mathrm{B}}$. It agrees with the expected $\mu_{\text {eff }}$ in the range $3.0-3.5 \mu_{\mathrm{B}}$ for dilute $\mathrm{Ni}^{2+}(S=1)$ systems and with the EHCF result concerning its ground-state spin, too. It also agrees with the estimate of the spin-orbit contribution to the effective momentum by eq. 4.82 [25] upon using the approximate transition energy of $7000 \mathrm{~cm}^{-1}$ (Table 1) and a spin-orbit coupling constant $\zeta=600 \mathrm{~cm}^{-1}$ which together arrive at $3.28 \mu_{\mathrm{B}}$. In $\mathrm{Co}(\mathrm{NCNH})_{2}$ the reciprocal magnetic susceptibility above the Curie temperature is not that perfectly linear as in $\mathrm{Ni}(\mathrm{NCNH})_{2}$ but the deviation form linearity does not prevent to determine the high-temperature effective magnetic momentum. It equals $4.52 \mu_{\mathrm{B}}$, in agreement with the spin-orbit enhanced value characteristic for the isolated $\mathrm{Co}^{2+}$ ions $(S=3 / 2)$ and the expectations from the EHCF-derived spin ground state. Note that calculations targeted at the precise amount of the spin-orbit enhancement of the effective momentum (as performed for $\mathrm{CoNCN}$ ) are impossible due to the lower symmetry of the protonated material.

Staying in the framework of the chemical paradigm, i.e., considering the effect of the protonation of the $\mathrm{NCN}^{2-}$ bridges on the spectral and magnetic characteristics, the protonation further lowers the $d$-shell chromophore symmetry by introducing nonequivalence 
among the nitrogen donor atoms. Among six donor nitrogens in the approximately octahedral arrangement around the metal ions (see Figure 2), just four in the equatorial plane are protonated. Thus, one may expect the splitting of the approximately octahedral many-electron terms of the respective $d$-shells to be somewhat stronger than in the MNCN series. The most striking difference is the change of the sequence of the excited states taking place in the pair of the iron compounds. In both, the lowest excitation is the spin-allowed ${ }^{5} T_{2} \rightarrow{ }^{5} E$ transition, which is split much more strongly $\left(3000 \mathrm{~cm}^{-1}\right)$ in the protonated compound than in the nonprotonated FeNCN (ca. $1000 \mathrm{~cm}^{-1}$ ). The next two excitations exchange their positions on the energy scale under protonation: in FeNCN, the second and third excited manifolds are ${ }^{3} T$ and ${ }^{3} E$, respectively, whereas in the protonated chromophore of $\mathrm{Fe}(\mathrm{NCNH})_{2}$ this order is inverted.

The patterns of the superexchange paths in the $M N C N$ series have already been established [5]. In agreement with prior work [27] the nearest-neighbor exchange within the metal layers (Figure 1) as mediated by the $\mu^{3}$-nitrogen atoms of the $\mathrm{NCN}^{2-}$ units has a ferromagnetic sign. By contrast, the interlayer (kinetic) superexchange, possibly mediated by the $\pi$-states of the $\mathrm{NCN}^{2-}$ units, has an antiferromagnetic sign. Addressing the possible superexchange paths in the $M(\mathrm{NCNH})_{2}$ series, the protonation significantly changes the coordination pattern of the $\mathrm{NCN}^{2-}$ units: instead of trigonal prismatic coordination characteristic for the [NiAs] structure type of $M \mathrm{NCN}$ providing $\mu^{3}$-nitrogens, UNCLEAR FORMULATION the protonated nitrogen atoms located in the equatorial planes of the $M \mathrm{~N}_{6}$ octahedra serve as only $\mu^{2}$-bridges. Like this it it shorter, and hopefully more clear This provides a superexchange path for ferromagnetic coupling between the metal ions in the $c$-direction (two neighbors for each metal ion). Following [27] the conjecture is that the magnitude of the $\mu$-nitrogen mediated ferromagnetic exchange slightly decreases in the hydrocyanamides as compared to the corresponding carbodiimides since the $M-\mathrm{N}-M$ angles controlling this quantity increase slightly but systematically while going from $M \mathrm{NCN}$ to $M(\mathrm{NCNH})_{2}: 95.9^{\circ} \rightarrow 98.5^{\circ}(\mathrm{Fe}) ; 95.7^{\circ} \rightarrow 100.0^{\circ}(\mathrm{Co}) ; 96.1^{\circ} \rightarrow 98.4^{\circ}$ (Ni). The paths extended through the $\mathrm{NCNH}^{-}$units themselves rather than through the protonated $\mu^{2}$-nitrogens must be assumed to be ferromagnetic in order to conform with the overall ferromagnetic ordering in the $M(\mathrm{NCNH})_{2}$ series. Otherwise, the ferromagnetic order would exist only in one-dimensional chains along the $c$-axes. Remarkably enough, the number of the nearest neighbors of each metal ion interacting though the $\mathrm{NCNH}^{-}$ units equals eight so that in respect of this type of interaction the metal ions effectively 
form a body-centered tetragonal lattice: each of the four $\mathrm{NCNH}^{-}$units coordinating a given metal ion through the protonated nitrogen atom couples to one other metal ion whereas each of two $\mathrm{NCNH}^{-}$units coordinating the given metal ion through the nonprotonated hydrogen couples to two other metal ions through its protonated end as shown in Figure 2.

To conclude the discussion we notice that, quite often, reproducing the size of the optical gap and magnetic moments in solid transition-metal compounds is considered as a crucial evidence for the strength of DFT in this area. A real problem persists, however. Even if density-functional theory were able to yield a qualitatively correct answer in terms of energy gaps in all cases (like it does for oxides, or NiNCN, or CoNCN, but not for FeNCN), it would not provide any reasonable description of the optical excitations. Since DFT operates by filled or empty electronic bands, an insulator's optical spectrum must then consist of a wide band with an edge at the energy of the gap. This picture is, however, in contradiction with experiment because the optical spectra of most transition-metal compounds follow completely different patterns: their "low-energy" parts consist of numerous lines of moderate width and do not noticeably differ from those of transition-metal complexes in solutions or from the transition-metal impurity ions in var-

ious hosts $[12,13]$. Such a situation definitely calls for an alternative treatment of the optical excitations of solid transition-metal compounds similar to materials at hand that would be able to reproduce the mentioned qualitative features of their behavior.

\section{Conclusion}

By using the many-particle local correlated EHCF technique we unequivocally establish the nature of the ground and low-energy excited states in the series of transition-metal carbodiimides $M \mathrm{NCN}$ with $M=\mathrm{Fe}-\mathrm{Ni}$. In all cases the ground states correspond to the weak-field high-spin scenario, the magnetic moments being antiferromagnetically coupled. The sequence of the excited states clearly corresponds to the pattern given by the phenomenological crystal-field theory. The predicted transition energies conform with the positions of the absorption bands in the near infrared and in the visible part of the spectrum. The pathological case of FeNCN as seen by DFT-based methods smoothly fits into the picture of the crystal-field insulators being similar to other members (MnNCN, CoNCN, and NiNCN). Analogously, for the $M(\mathrm{NCNH})_{2}$ series of compounds with $M=$ $\mathrm{Fe}-\mathrm{Ni}$ the high-spin ground states are established in agreement with available experi- 
ments. The existence of the absorption bands in the near infrared and visible part of the spectrum is confirmed.

\section{Acknowledgments}

This work has been performed with the support of Deutsche Forschungsgemeinschaft and the Excellence Initiative of the German federal and state governments. In addition, we acknowledge the Russian Foundation for Basic Research for the financial support dispatched to ALT through the grant No. 10-03-00155. The authors are thankful to Prof. I. Mayer for sending reprints of his works.

\section{References}

[1] C. Janiak, Dalton Trans. 2003, 2781.

[2] X.-H. Liu, M. Krott, P. Müller, C. H. Hu, H. Lueken, R. Dronskowski, Inorg. Chem. 2005, 44, 3001 .

[3] M. Krott, X. H. Liu, B. P. T. Fokwa, M. Speldrich, H. Lueken, R. Dronskowski, Inorg. Chem. 2007, 46, 2204.

[4] X.-H. Liu, L. Stork, M. Speldrich, H. Lueken, R. Dronskowski, Chem. Eur. J. 2009, 15,1558

[5] H. Xiang, R. Dronskowski, B. Eck, A. L. Tchougréeff, J. Phys. Chem. A 2010, 114, 12345.

[6] V. I. Anisimov, J. Zaanen, O. K. Andersen, Phys. Rev. B 1991, 44, 943.

[7] A. Georges, G. Kotliar, W. Krauth, M. J. Rozenberg, Rev. Mod. Phys. 1996, 68, 13; K. Held, I. A. Nekrasov, G. Keller, V. Eyert, N. Blümer, A. K. McMahan, R. T. Scalettar, T. Pruschke, V. I. Anisimov, D. Vollhardt, Quantum Simulations of Complex Many-Body Systems: From Theory to Algorithms, Lecture Notes, J. Grotendorst, D. Marx, A. Muramatsu (Eds.), John von Neumann Institute for Computing, Jülich, NIC Series 2002, 10, 175.

[8] A. V. Soudackov, A. L. Tchougréeff, I. A. Misurkin, Theor. Chim. Acta 1992, 83, 389. 
[9] X.-H. Liu, R. Dronskowski, R. Glaum, A. L. Tchougréeff, Z. Allg. Anorg. Chem. 2010, 636, 343.

[10] M. Krott, X. Liu, P. Müller, R. Dronskowski, J. Solid State Chem. 2007, 180, 307.

[11] W. A. Harrison, Electronic Structure and the Properties of Solids, Freeman, San Francisco 1990 (Chapter 19).

[12] A. B. P. Lever, Inorganic Electronic Spectroscopy, Elsevier, Amsterdam 1984;

[13] C. A. Morrison, Crystal Fields for Transition-Metal Ions in Laser-Host Materials, Springer, Berlin 1992.

[14] A. L. Tchougréeff, R. Dronskowski, Int. J. Quant. Chem. 2009, 109, 2606.

[15] A. V. Soudackov, A. L. Tchougréeff, I. A. Misurkin, Int. J. Quant. Chem. 1996, 58, 161.

[16] A. V. Soudackov, A. L. Tchougréeff, I. A. Misurkin, Int. J. Quant. Chem. 1996, 5\%, 663.

[17] A. V. Sinitsky, M. B. Darkhovskii, A. L. Tchougréeff, I. A. Misurkin, Int. J. Quant. Chem. 2002, 88, 370.

[18] C. E. Schäffer, C. K. Jørgensen, Mol. Phys. 1965, 9, 401.

[19] A. V. Soudackov, A. L. Tchougréeff, I. A. Misurkin, Zh. Fiz. Khim. 1994, 68, 1256.

[20] A. V. Soudackov, A. L. Tchougréeff, I. A. Misurkin, Zh. Fiz. Khim. 1994, 68, 1264.

[21] M. G. Razumov, A. L. Tchougréeff, Zh. Fiz. Khim. 2000, 74, 87.

[22] A. M. Tokmachev, A. L. Tchougréeff, J. Solid State Chem. 2003, 176, 633.

[23] T. C. Ozawa, S. J. Kang, J. Appl. Cryst. 2004, 37, 679.

[24] X. Tang, R. Dronskowski, in preparation.

[25] H. Lueken, Magnetochemie; Teubner: Stuttgart, Germany, 1999.

[26] E. König, S. Kremer, Magnetism Diagrams for Transition Metal Ions; Plenum Press: New York, 1979, p. 260. 
[27] M. A. Atanasov, S. Angelov, I. Mayer, Sol. St. Commun. 1985, 56, 743; M. A. Atanasov, S. Angelov, I. Mayer, J. Mol. Struct. (THEOCHEM) 1989, 187, 23. 
Table 1: $d$ - $d$ transition energies for the $M \mathrm{NCN}$ and $M(\mathrm{HNCN})_{2}$ series of materials. $B$ and $C$ stand for the Racah parameters $\left(\mathrm{cm}^{-1}\right)$ used for calculating electron-electron interactions in the respective $d$-shells.

\begin{tabular}{|c|c|c|c|}
\hline & $E^{\text {theor }}\left(\mathrm{cm}^{-1}\right)$ & & $E^{\text {theor }}\left(\mathrm{cm}^{-1}\right)$ \\
\hline \multicolumn{2}{|c|}{ FeNCN } & \multicolumn{2}{|c|}{$\mathrm{Fe}(\mathrm{NCNH})_{2}$} \\
\hline \multicolumn{4}{|c|}{$B=920 ; \quad C=4040$} \\
\hline \multirow[t]{2}{*}{${ }^{5} T_{2} \rightarrow{ }^{5} E$} & 6742 & ${ }^{5} T_{2} \rightarrow{ }^{5} E$ & 6070 \\
\hline & 7684 & & 9110 \\
\hline \multirow[t]{3}{*}{$\rightarrow{ }^{3} \mathrm{~T}$} & 15579 & $\rightarrow{ }^{3} E$ & 14439 \\
\hline & 15721 & & 14828 \\
\hline & 15964 & $\rightarrow{ }^{3} T$ & 16172 \\
\hline \multirow[t]{2}{*}{$\rightarrow{ }^{3} E$} & 18169 & & 17102 \\
\hline & 18388 & & 18386 \\
\hline$\rightarrow{ }^{1} A_{1}$ & 19860 & $\rightarrow{ }^{1} A_{1}$ & 18838 \\
\hline \multicolumn{2}{|c|}{$\mathrm{CoNCN}$} & \multicolumn{2}{|c|}{$\mathrm{Co}(\mathrm{NCNH})_{2}$} \\
\hline \multicolumn{4}{|c|}{$B=970 ; C=4500$} \\
\hline \multirow[t]{3}{*}{${ }^{4} T_{1} \rightarrow{ }^{4} T_{2}$} & 5696 & ${ }^{4} T_{1} \rightarrow{ }^{4} T_{2}$ & 5901 \\
\hline & 5826 & & 5980 \\
\hline & 5861 & & 6127 \\
\hline$\rightarrow{ }^{4} A_{2}$ & 11909 & $\rightarrow{ }^{4} A_{2}$ & 12464 \\
\hline \multirow[t]{2}{*}{$\rightarrow{ }^{2} E$} & 14611 & $\rightarrow{ }^{2} E$ & 13928 \\
\hline & 14653 & & 14457 \\
\hline \multirow[t]{3}{*}{$\rightarrow{ }^{2} T$} & 18938 & $\rightarrow{ }^{2} T$ & 18780 \\
\hline & 19102 & & 18864 \\
\hline & 19113 & & 18969 \\
\hline$\rightarrow{ }^{2} A$ & 19268 & $\rightarrow{ }^{2} A$ & 19058 \\
\hline \multicolumn{2}{|c|}{ NiNCN } & \multicolumn{2}{|c|}{$\mathrm{Ni}(\mathrm{NCNH})_{2}$} \\
\hline \multicolumn{4}{|c|}{$B=1030 ; C=4860$} \\
\hline \multirow[t]{3}{*}{${ }^{3} A_{2} \rightarrow{ }^{3} T$} & 5856 & ${ }^{3} A_{2} \rightarrow{ }^{3} T$ & 5947 \\
\hline & 5977 & & 6785 \\
\hline & 7042 & & 6860 \\
\hline \multirow[t]{3}{*}{$\rightarrow{ }^{3} \mathrm{~T}$} & 10223 & $\rightarrow{ }^{3} T$ & 10738 \\
\hline & 10730 & & 11359 \\
\hline & 11940 & & 11618 \\
\hline \multirow[t]{2}{*}{$\rightarrow{ }^{1} E$} & 16613 & $\rightarrow{ }^{1} E$ & 16991 \\
\hline & 17046 & & 17087 \\
\hline
\end{tabular}




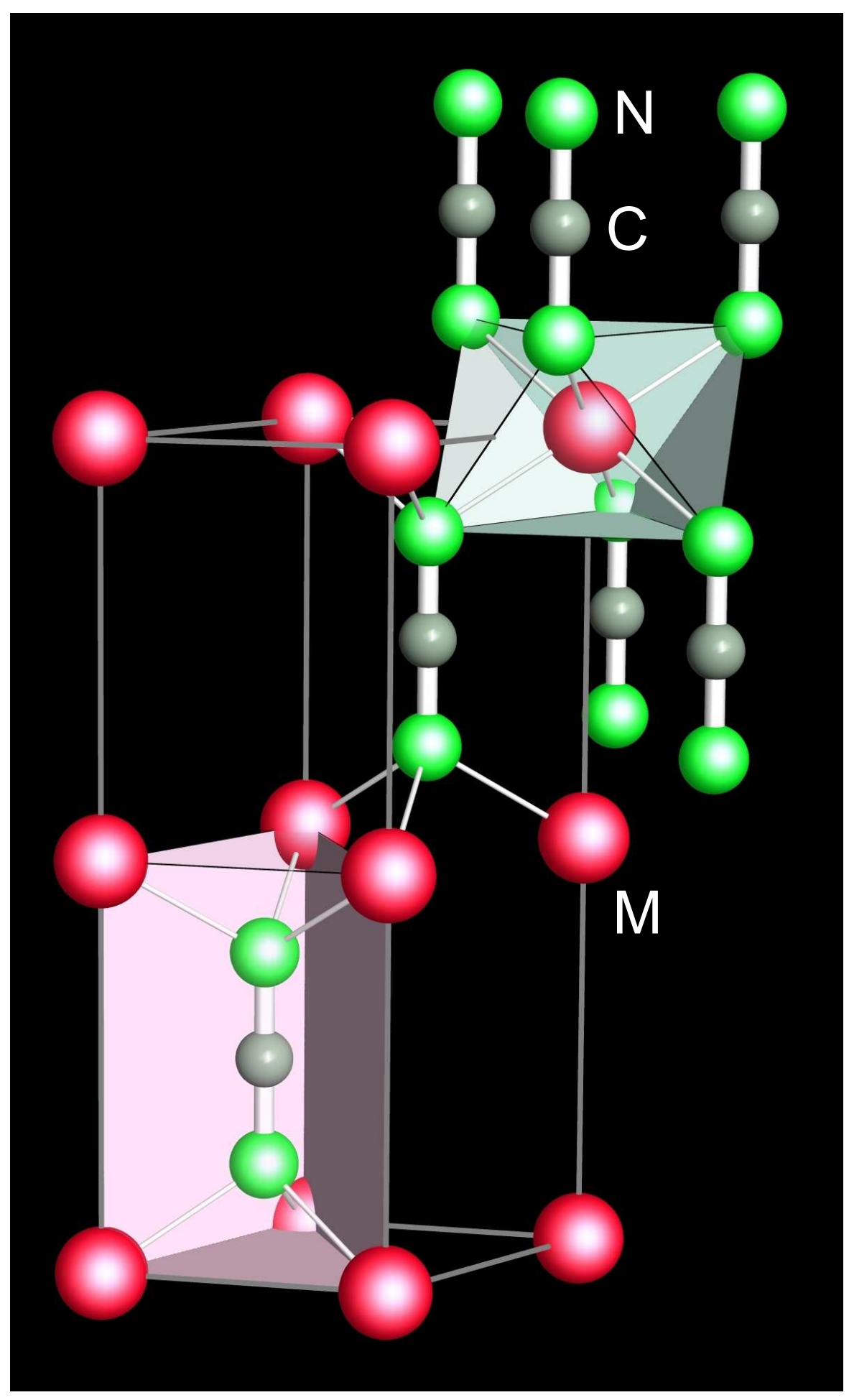

Figure 1: Perspective view [23] into the crystal structure of MNCN compounds with an approximately octahedral coordination of the (red) metal cation and a trigonal-prismatic one for the carbodiimide unit; $\mathrm{N}$ atoms in green, $\mathrm{C}$ atoms in grey. 


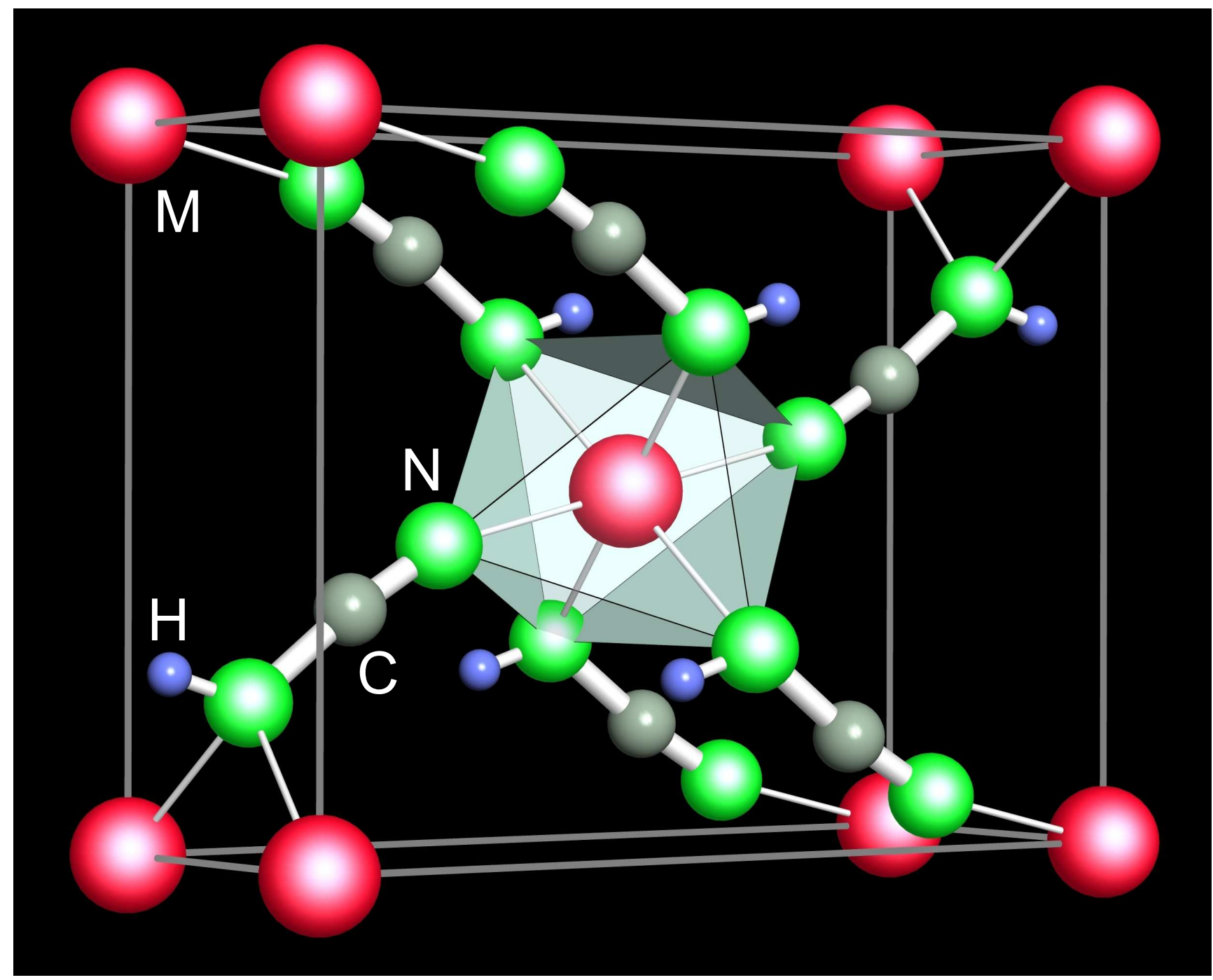

Figure 2: Perspective view [23] into the crystal structure of $M(\mathrm{HNCN})_{2}$ compounds with an approximately octahedral coordination of the (red) metal cation by (green) $\mathrm{N}$ atoms of the protonated carbodiimide units; $\mathrm{C}$ atoms in grey, $\mathrm{H}$ atoms in blue. 